\title{
Formação em Psicologia para a Atenção Básica à Saúde e a Integração Universidade-Serviço- Comunidade
}

Formación En Psicología Para Atención Básica A La Salud Y La Integración Universidad-Servicio-Comunidad

Psychology Education For Basic Health Care And University-Service-Community Integration

Monica Lima,

Manuela Brito \& Alice Firmino

Universidade Federal da Bahia
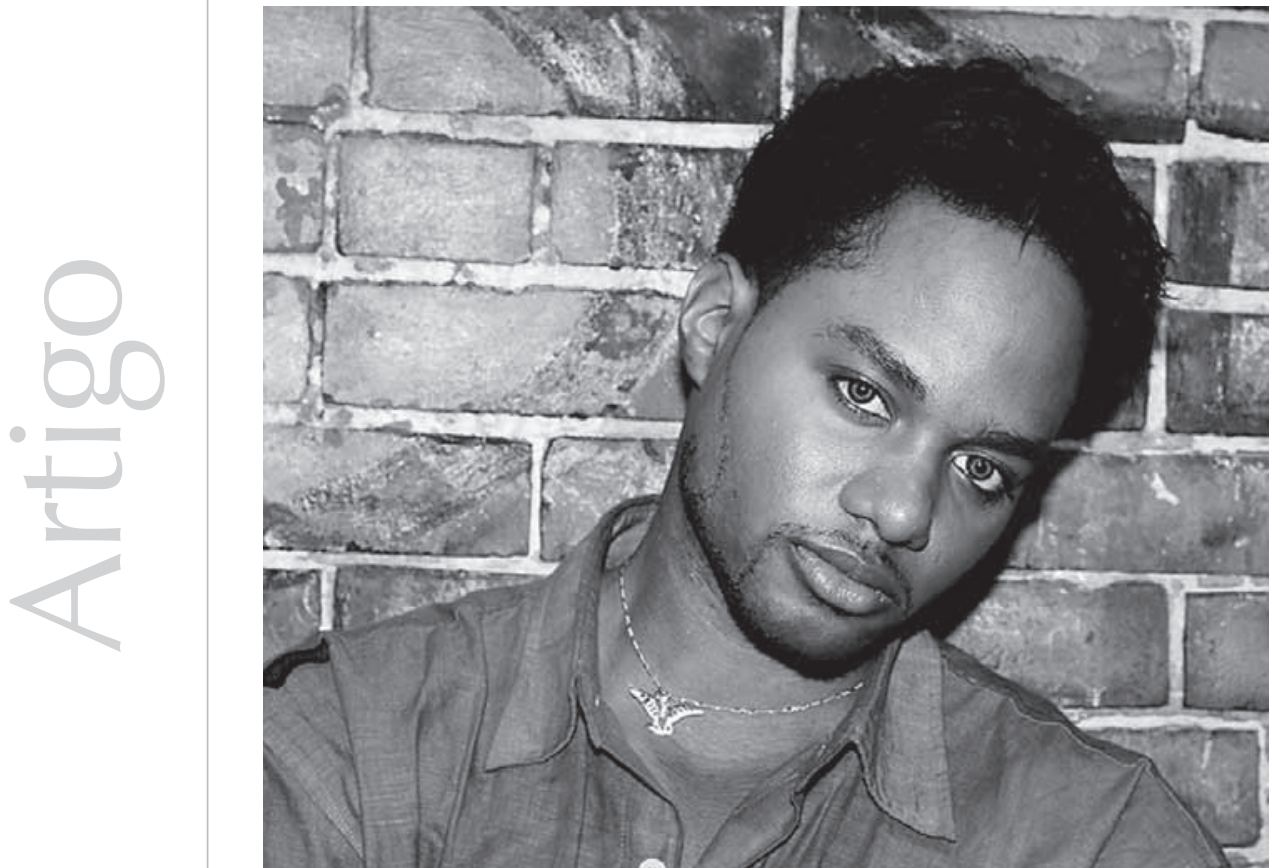
Resumo: O Integrar/SUS-Educação é um projeto que articula ensino e extensão no campo da saúde pública/ coletiva para a formação de estudantes de Psicologia, na perspectiva da integração universidade-serviçocomunidade, em Salvador-Bahia. A parceria com o Centro Saúde São Francisco (SMS-Salvador) e com o Curso de Psicologia (IPS/UFBA) conjuga duas motivações: 1) garantir a inserção dos estudantes em contextos de trabalho desde a graduação no campo da saúde coletiva/pública, particularmente na atenção básica à saúde, e 2) otimizar os recursos para a consolidação do programa Saúde e Prevenção nas Escolas, com o foco em DST/HIV/AIDS. O presente projeto capacitou 308 graduandos, realizou 149 oficinas Árvore dos Prazeres, envolveu 4.550 jovens e distribuiu 8.500 preservativos em 15 escolas públicas de Salvador, entre 2007 a 2011. Essa experiência tem favorecido o processo ensino-aprendizagem, desenvolvendo habilidades para fazeres na atenção básica, e potencializado o CS - SF como serviço de referência para os jovens e graduandos de Psicologia.

Palavras-chave: Formação do psicólogo. Política de saúde. HIV-AIDS. Estudantes universitários

Abstract: Integration/SUS-Education is a project that articulates education and experience in the field of public/collective health for the education of psychology students from the perspective of the integration university - service - community in Salvador, Bahia. The partnership with the San Francisco Health Center (SMS-Salvador) and the IPS/UFBA Psychology Faculty unites two incentives: 1. guarantee the insertion of students in work contexts as they graduate in the field of public/collective health, particularly in the basic health care and 2. optimize resources to strengthen the school program Health and Prevention, with a focus on DST/HIV/AIDS. The present project trained 308 undergraduation students, realized 149 workshops Tree of Pleasures, involved 4.550 young people, and distributed 8.500 condoms in 15 public schools in Salvador between 2007 and 2011. This process has contributed to the teaching-learning process developing abilities geared toward basic health care and illustrating the potential of the CS - SF as a reference service for youngsters and for psychology students.

Keywords: Psychologist education. Health care policy. Human immunodeficiency virus. College students.

Resumen: El Integrar/SUS-Educación es un proyecto que articula enseñanza y extensión en el campo de la salud pública/colectiva para la formación de estudiantes de psicología, en la perspectiva de la integración universidad-servicio-comunidad, en Salvador - Bahia. La aparcera del Centro de Salud São Francisco (SMS - Salvador) y del Curso de Psicología (IPS/UFBA) conjuga dos motivaciones: 1) garantizar la inserción de los estudiantes en contextos de trabajo desde la graduación en el campo de la salud pública/colectiva, particularmente en la atención básica a la salud; 2) optimizar los recursos para la consolidación del programa Salud y Prevención en las Escuelas, con el foco en DST/VIH/SIDA. El presente proyecto ha capacitado 308 estudiantes, ha llevado a cabo 149 talleres "Árbol de los Placeres", ha tenido la participación de 4550 jóvenes, ha repartido 8500 preservativos en 15 escuelas públicas de Salvador, entre 2007 y 2011. Esta experiencia ha favorecido el proceso enseñanza-aprendizaje, desarrollando habilidades para quehaceres en la atención básica y potenciando el CS-SF como servicio de referencia para los jóvenes y estudiantes de psicología.

Palabras-clave: Formación del psicologo. Política pública de salud. VIH - SIDA. Estudiantes universitários.

Este artigo tem como objetivo relatar o processo de implantação e consolidação do Projeto Integrar SUS-Educação nos últimos três anos e meio. Esse projeto operacionaliza a tríade ensino-serviço-comunidade, desde 2007, através da oferta regular da disciplina Psicologia da Saúde, oferecida pelo Instituto de Psicologia (IPS/UFBA) no cenário de prática-aprendizagem do Centro de Saúde São Francisco da Secretária Municipal de Saúde de Salvador-Bahia (SMS) e de escolas públicas do referido Município.
Nesta comunicação, apresentam-se as atividades propostas para a formação de estudantes do Curso de Progressão Linear de Psicologia (CPL - Psico - IPS/UFBA) e, mais recentemente, dos estudantes dos Bacharelados Interdisciplinares de Humanidades e de Saúde do Instituto de Humanidades, Artes e Ciências (IHAC/ UFBA), os resultados alcançados, os desafios e as perspectivas para a qualificação de profissionais para a consolidação do Sistema Único de Saúde (SUS) no eixo da educação para a saúde. 
A ampliação da atividade de psicólogos no campo da saúde pública, antes circunscrita a serviços especializados de saúde mental, é considerada emergente no Brasil. Essa expansão, decorrente da saída dos psicólogos da clínica privada para a área de saúde pública, tem como impulsionador um amplo movimento de forças sociais, que envolve profissionais de saúde e usuários interessados em discussões sobre as condições de saúde da população, consolidando-se em propostas concretas nas últimas Conferências Nacionais de Saúde e nas específicas de saúde mental. Um importante aspecto dessa expansão refere-se à política de recursos humanos em saúde, que incentiva a formação de equipes multidisciplinares (Silva, 1992; Lo Bianco, Bastos, Nunes, \& Silva, 1994; Bastos \& Achcar, 1994; Dimenstein, 1998; Carvalho \& Yamamoto, 1999).

A maioria dos psicólogos lotados, em 2005, em unidades básicas de saúde (UBS) de Salvador entrou, na década de 90, através de concurso público (Lima, 2005a). A entrada de psicólogos nas UBSs é proveniente da ampliação da assistência à saúde mental na atenção básica (Dimenstein, 1998; Carvalho \& Yamamoto, 1999), que, em Salvador, pode ser considerada não só tardia como incipiente. Em 2002, contava-se com apenas seis psicólogos em cinco UBSs, e, em 2003, apenas com quatro, distribuídos em quatro distritos sanitários dos doze existentes na capital (Lima, 2005a). Segundo levantamento da Coordenadoria de Desenvolvimento de Recursos Humanos (CDRH - SMS), em 2010, havia 107 profissionais de Psicologia vinculados à SMS: 58,8 \% deles atuando em CAPS e 16,8\%, na atenção básica; os demais estavam distribuídos em coordenações dos distritos ou aguardando que fosse definida sua lotação na rede.

Estudos importantes, desde o início da década de 90, analisam os desafios e as perspectivas para a formação de psicólogos na saúde pública no processo de implantação e de consolidação do SUS, bem como a necessária articulação das ações intersetoriais para lidar com necessidades de saúde pertinentes aos setores da saúde e da educação (Boarini, 1993). Segundo Boarini, na saúde pública/ coletiva, deve-se operar na articulação indivíduo, sociedade e serviço, que busca materializar-se em ações adequadas às particularidades das situações de trabalho, naquelas que vençam as fragilidades dos conhecimentos aprendidos nos espaços acadêmicos e que coloquem em xeque muitas das tradicionais intervenções psicológicas incansavelmente oferecidas.

Pode-se considerar que o psicólogo, nas unidades básicas de saúde - UBS de Salvadorainda é um profissional estranho, e, de modo geral, ainda precisa aprender a cultura local. Por um lado, um novo sistema cultural se apresenta, em que procedimentos comuns aos serviços públicos de saúde se tornam complexos aos seus olhos e ouvidos. Sua bagagem parece pesada e pouco adequada a esse novo espaço de trabalho: os psicólogos tendem a reproduzir o modelo clássico da Psicologia clínica que aprenderam durante a graduação, que dá o tom, o compasso e a forma de atuarem (Lima, 2005a). Por outro lado, os demais profissionais esperam que o psicólogo, mesmo na atenção básica de saúde, organize seu trabalho a partir da ênfase na marcação de consultas individuais que caracterizam a atuação mais difundida em Psicologia clínica (Lima, 2005a, 2005b).

Em 2006, Spink (2007) ressaltou que havia em torno de $15 \%$ do total de psicólogos trabalhando no SUS, mais ou menos 22.500 trabalhadores, no Brasil. Certamente, hoje essa porcentagem aumentou, mas seguindo o raciocínio de que se formam 17 mil psicólogos por ano, no Brasil, parcela generosa dela irá pleitear, ao menos, um dos seus empregos no SUS, considerando a dupla inserção do mercado de trabalho que persiste na 
Psicologia. Esses futuros trabalhadores precisam de formação compatível com a consolidação do SUS, desde a graduação. Uma das exigências será a participação em equipes de trabalho nos níveis primário, secundário e terciário de assistência à saúde. Almeja-se que essa atuação se dê em uma perspectiva interdisciplinar, em vários âmbitos de trabalho, do atendimento direto a população à gestão de pessoas.

Considerando a faixa de trabalhadores de Psicologia nas organizações públicas, Macedo, Heloani e Cassilato (2010) destacam que a maioria dos psicólogos está alocada no nível de administração municipal (45,9\%), o que se deve à inserção do psicólogo em equipes da área de saúde, em hospitais ou em outras unidades do sistema público de saúde. As atividades mais empregadas pelos psicólogos também ressaltam a sua presença na área da saúde: 32\% aplicam testes psicológicos, 29,6\% fazem psicodiagnóstico, além do atendimento de crianças com transtornos de aprendizagem (20\%), também desenvolvido no setor público; além disso, 16,8\% destes dão assistência psicológica a pacientes clínicos e cirúrgicos. Os psicólogos são trabalhadores assalariados - 19,9\% possuem apenas um vínculo empregatício com o setor público, $11,3 \%$, no setor privado, e 3,7\%, no terceiro setor. Apenas $17,6 \%$ deles têm o trabalho autônomo como principal vínculo. Para a grande maioria, o trabalho autônomo é complementar aos diversos tipos de trabalho assalariado, sendo característico da profissão o fato de psicólogos trabalharem em mais de um local: 2/3 dos psicólogos empregados têm mais de um emprego, $33,6 \%$ do total de respondentes têm duas inserções e 31,5\% têm mais de duas inserções no mercado de trabalho.

Considerando essa realidade, descrita brevemente, justifica-se o empenho para a implantação de projetos de extensão educativos que impulsionem a formação extramuro universitário qualificando os psicólogos para atuar no SUS. Além disso, é preciso "aceitar que precisamos nos aproximar da população 'não branca' (ou embranquecida), não elitista, menos abastada, não letrada [...] e das problemáticas decorrentes da desigualdade social, da discriminação racial, da falta de direitos humanos, etc" (Lima, 2010, p. 12). Certamente, é preciso renovar mais do que a matriz curricular e atingir a organização do processo de trabalho dos envolvidos nessa árdua e fascinante tarefa. No final da década de 90, Boarini (1996, p. 129) já apontava:

a necessidade de inserir no currículo do curso de psicologia disciplinas que abordem a saúde pública: inclusão do estágio em UBS como fonte enriquecedora da formação profissional; a necessidade de se criarem situações que possibilitem o trabalho multiprofissional em que todos os aspectos devam estar sob a responsabilidade de professores com experiência na área

É disso que trata este artigo, de um dos pontos de partida para a formação de psicólogos para contextos de trabalho por área profissional ou multiprofissional em saúde. No CPL - Psico, os esforços nos últimos anos têm sido no sentido de oferecer um componente obrigatório que proporcione a todos os estudantes a inserção qualificada e mais precoce no SUS, buscando superar a visão superficial e acrítica sobre o sistema público de saúde. Além disso, busca-se garantir atividades práticas que oportunizem o desenvolvimento de habilidades e competências adequadas para a inserção na atenção primária à saúde. Nessa direção, o CLP - Psico aderiu ao Programa de Reorientação da Formação de Profissionais de Saúde (Pró-Saúde) e ao Programa de Educação pelo Trabalho da Saúde da Família (PET-SF), além de oferecer regularmente disciplinas na interface Psicologia e saúde pública/coletiva.

Segundo o Centro de Referência em Políticas Públicas e Psicologia (CREPOP), os psicólogos 
têm se inserido cada vez mais em atuações que remetem à implementação ou à consolidação de políticas públicas de saúde, entre elas aquela relativa à política de combate ao HIV/ AIDS (CREPOP, 2008).

Justificam-se, assim, as práticas profissionalizantes que possam aumentar a capacidade desses profissionais para o trabalho nesse âmbito. Por outro lado, a tendência da epidemia de HIV/AIDS demanda uma atenção maior para a população entre 17 e 20 anos de idade, cuja prevalência passou de 0,09\% para 0,12\% nos últimos cinco anos; além disso, quanto menor a escolaridade, maior o percentual de infectados pelo HIV (prevalência de 0,17\% entre aqueles com ensino fundamental incompleto e de $0,10 \%$ entre os que têm ensino fundamental completo) (Brasil, 2010).

De acordo com a pesquisa de Comportamento, Atitudes e Práticas da População Brasileira (Departamento de DST/AIDS e Hepatites Virais, 2008), 97\% dos jovens de 15 a 24 anos de idade sabem que o preservativo é a melhor maneira de evitar a infecção pelo HIV, mas o uso cai à medida que a parceria sexual se torna estável. O percentual de uso do preservativo na primeira relação sexual é de $61 \%$, e chega a $30,7 \%$ em todas as relações com parceiros fixos. Esses dados reforçam a necessidade de parceria entre universidadeserviço-comunidade com iniciativas que aproximem metodologias ativas para a abordagem dos jovens em relação a essa temática.

\section{Método de trabalho: integração ensino-serviço-comunidade}

A disciplina Psicologia da Saúde tem uma carga horária de 68 horas semestrais (34 de atividades práticas e 34 horas teóricas). Esse componente curricular tem por objetivos: 1) compreender a relação da Psicologia com o campo da saúde pública/coletiva como ciência e profissão, 2) apresentar e refletir criticamente sobre as políticas públicas de saúde vigentes no Brasil, 3) identificar os desafios decorrentes da inserção dos psicólogos no sistema de saúde vigente no Brasil e 4) apresentar os fundamentos e estratégias de prevenção, promoção e cuidado em saúde, destacando as contribuições da Psicologia para o enfrentamento de questões relativas à saúde pública/coletiva.

O principal cenário de prática-aprendizagem é o Centro de Saúde São Francisco (CS SF) do Distrito Sanitário Centro Histórico $(\mathrm{DSCH})$ de Salvador-Bahia. Esse centro pode ser considerado misto, porque oferece serviços de atenção básica como imunização, assistência de enfermagem, serviço social, odontologia, farmácia, psicologia e de atenção especializada em DST/AIDS, como consultas de infectologia, testagem para HIV, VDRL (sífilis) e hepatite $\mathrm{B}$, com aconselhamento pré e pós-teste. O centro desenvolve programas específicos, tais como: controle do tabagismo, controle de tuberculose, saúde e prevenção nas escolas, prevenção das DST/HIV/AIDS junto a crianças, adolescentes e jovens em situação de rua. Essas ações de saúde ocorrem na unidade e na comunidade a partir das demandas apresentadas. O DSCH tem como aparelhos de serviços de saúde: cinco unidades básicas de saúde, um centro de atenção psicossocial - CAPS, um Programa de Agente Comunitário de Saúde, duas unidades do Programa de Saúde da Família, um centro de atenção especializada, um centro de referência da saúde do trabalhador e um centro especializado odontológico.

O CS - SF está vinculado à Coordenação Municipal de DST/AIDS na área de assistência e prevenção às DST/HIV/AIDS, atuando na defesa dos direitos humanos e dos direitos de cidadania, contra o preconceito e a discriminação e na garantia do acesso universal à assistência gratuita (incluindo medicamentos específicos). O CS - SF conta 
com equipe multiprofissional composta por: médicos equipes de enfermagem, assistentes sociais, psicólogo, farmacêutico e dentista, além de uma equipe de apoio administrativo que se apresenta de forma importante e estratégica no processo de acolhimento dos usuários que procuram os serviços.

A parte prática da disciplina Psicologia da Saúde é organizada em três eixos; um deles se desenvolve na sala de aula, e os outros dois eixos, no CS - SF ou nos Centros de Testagem e Aconselhamento da rede municipal de saúde de Salvador; são eles: 1) análise de situações-problema passíveis de serem encontrados nos serviços públicos de saúde em rodas de conversa e simulação de ações concretas do cotidiano do trabalho reproduzíveis em sala de aula, como o uso de metodologias ativas, 2) entrada do graduando como usuário direto do SUS, 3) capacitação dos graduandos para exercerem o papel de facilitadores da oficina Árvore dos Prazeres, estratégia prevista no programa Saúde e Prevenção nas Escolas: Atitude pra Curtir a Vida (Brasil, 2005).

\section{Simulações na sala de aula}

Em relação ao primeiro eixo, trata-se de oferecer atividades de simulação com o objetivo de preparar os discentes no exercício de tarefas realizadas na atenção básica à saúde, como, por exemplo: a) preenchimento e análise do protocolo de coleta de dados sobre a vida sexo-afetiva de usuários no aconselhamento pré-teste individual em DST/HIV/AIDS e b) entrega de resultados no aconselhamento pós-teste com prévia indicação de literatura científica atualizada sobre a epidemia de HIV/AIDS nas dimensões de prevenção, promoção e cuidado de pessoas que vivem com HIV/ AIDS.

\section{Entrada como usuário no SUS: o que aprendi com isso para a atuação profissional?}

Nessa etapa, os estudantes vão ao CS SF ou a um dos Centros de Testagem e Aconselhamento da rede municipal pública de saúde, na condição de usuários, em demanda espontânea para participar dos aconselhamentos coletivo e individual (préteste). A participação no aconselhamento pós-teste não é uma exigência dessa prática, por questões éticas, para evitar constrangimentos e assegurar o direito do usuário-cidadão no que diz respeito à sua decisão ou não de conhecer a sorologia para o HIV. A experiência de estar usuário - SUS no nível primário de atenção à saúde busca familiarizar os estudantes com os seguintes pressupostos: a inserção do aconselhamento e do diagnóstico do HIV na rotina dos serviços da rede básica de saúde 1) implica uma reorganização do processo de trabalho da equipe e do serviço como um todo, 2) requer uma atenção para o tempo de atendimento, reformulações de fluxo da demanda, funções e oferta de atividades no serviço, 3) estimula mudanças de valores e práticas e exige uma preparação da equipe/serviço para acolher os processos subjetivos dos usuários, 4) é parte essencial dessa prática conhecer as principais vulnerabilidades para a infecção do HIV, as necessidades particulares dos usuários, suas características e estilos de vida e desenvolver uma abordagem sobre os riscos, respeitando as suas especificidades (Brasil, 2003).

A atividade inclui a entrega de um relato individual sobre essa experiência que esboce reflexões sobre a questão sou usuário SUS: o que aprendi com isso para a atuação profissional? Destaca-se o quão significativa tem sido a experiência como estudanteusuário a partir dos relatos dos discentes, que envolvem as dimensões subjetivas do estar estudante de Psicologia, mas também 
de estar usuário e de suas expectativas como futuros profissionais de saúde.

\section{Oficinas como método de intervenção psicossocial: integração universidade- serviço-comunidade}

De modo a operacionalizar esse eixo da prática da disciplina Psicologia da Saúde, no sentido de assegurar a aproximação qualificada dos estudantes com um dos fazeres dos profissionais de saúde no campo da saúde pública/coletiva, em acordo com a gestão da CS -SF, decidiu-se pela potencialização de um dos programas desenvolvidos na unidade com a colaboração direta da psicóloga no processo de ensinoaprendizagem dos discentes regularmente matriculados na referida disciplina. A escolha desse programa esteve orientada pelo fato de poder incluir um número significativo de graduandos em atividades desenvolvidas por psicólogos, sem causar transtorno na rotina da unidade, contribuindo para o aumento do Setor de Psicologia da unidade e proporcionando aos graduandos o desenvolvimento de habilidades que não eram reforçadas durante a graduação, como é o caso do papel de facilitador de oficinas em saúde ou trabalho em grupo em situações concretas de ensino-aprendizagem.

Além disso, o programa escolhido foi Saúde e Prevenção nas Escolas: Atitude pra Curtir a Vida, já implantado no CS - SF desde 2006, porque esse programa tem como objetivo central a promoção da saúde sexual e da saúde reprodutiva, visando a reduzir a vulnerabilidade de adolescentes e jovens às DST, à infecção pelo HIV, à AIDS e à gravidez não planejada por meio de ações nas escolas e nas unidades básicas de saúde (Brasil, 2005). O foco definido foi DST/HIV/AIDS, considerando a atuação do CS - SF como uma unidade de referência nessa temática.
Inicialmente, a psicóloga da CS - SF e a docente da IPS - UFBA realizam a oficina interna com os estudantes na sala de aula, de modo que eles conheçam os procedimentos e o modo de execução dessa oficina. Há também aula teórica sobre o que é uma oficina, como planejá-la e avaliá-la como um método de intervenção psicossocial (Afonso, Abade, Akerman, Coelho, Medrado, Paulino, \& Pimenta, 2003) e de conceitos como vulnerabilidade, diferenciando grupo de risco e comportamento de risco (Ayres, FrançaJunior, Calazans, \& Salletti-Filho, 2003).

As oficinas externas são realizadas em escolas públicas da rede municipal ou estadual de educação de Salvador. Os estudantes, em dupla ou em trio, após as atividades de preparação, são inseridos nas salas de aula do ensino médio e facilitam sozinhos as oficinas. A professora e a preceptora estão presentes na escola para organizar e dar suporte frente a qualquer necessidade. As oficinas são realizadas nas escolas, ao mesmo tempo, no dia e no turno do componente curricular Psicologia da Saúde. Elas acontecem, prioritariamente, em escolas do Distrito Centro Histórico, uma vez que o objetivo é tornar o CS - SF uma referência a que o jovem/adolescente possa recorrer sempre que necessitar. A oficina tem como foco a sexualidade e a infecção DST/HIV, é organizada em um único encontro de 1 hora e 30 minutos, para estudantes maiores de 14 anos, e é composta das seguintes etapas: 1) aquecimento, 2) levantamento e discussão dos seguintes temas-geradores: prazer, risco e proteção, 3) demonstração e distribuição de preservativos femininos e masculinos, 4) entrega do questionário de autoavaliação para a vulnerabilidade DST/HIV/AIDS, que é um instrumento em linguagem adequada para a população-alvo, 5) indicação de unidades de saúde com oferta para o aconselhamento pré e pós-teste, com distribuição de preservativos e 6) avaliação da oficina pelos participantes. 


\section{Integração universidade-serviço-comunidade: algumas conquistas}

No período de implantação e de consolidação desse projeto, capacitaram-se 308 graduandos CPL - Psico, entre eles, mais recentemente, alguns do BI de humanidades e de saúde, que realizaram 149 oficinas, atingindo 4.550 jovens estudantes, com distribuição de 8.500 preservativos masculinos e femininos (Tabela 01).

Tabela 1. Caracterização das oficinas realizadas entre 2007 a 2010 no Projeto Integrar SUS Educação

\begin{tabular}{lcccccccccc}
\hline Semestres & 2007.2 & 2008.1 & 2008.2 & 2009.1 & 2009.2 & 2010.1 & 2010.2 & 2011.1 & Total \\
\hline $\begin{array}{l}\text { Graduandos } \\
\text { de Psicologia }\end{array}$ & 30 & 40 & 40 & 40 & 40 & 40 & 40 & 38 & 308 \\
\hline $\begin{array}{l}\text { Estudantes do } \\
\text { ensino médio }\end{array}$ & 250 & 300 & 300 & 300 & 600 & 600 & 1000 & 900 & 4550 \\
\hline $\begin{array}{l}\text { Número de ofici- } \\
\text { nas realizadas }\end{array}$ & 10 & 12 & 12 & 30 & 30 & 30 & 40 & 36 & 149 \\
\hline $\begin{array}{l}\text { Número de } \\
\text { escolas } \\
\text { beneficiadas }\end{array}$ & 1 & 1 & 1 & 2 & 2 & 3 & 3 & 3 & 15 \\
\hline $\begin{array}{l}\text { Distribuição de } \\
\text { insumos } \\
\text { (preservativos) }\end{array}$ & 500 & 600 & 600 & 600 & 1200 & 1200 & 2000 & 1800 & 8500 \\
\hline
\end{tabular}

Fonte: Dados da pesquisa

Foi percebido que, após a realização das oficinas, principalmente em escolas no território do CS SF, há um aumento da frequência em 30\% de adolescentes/jovens à unidade. Espera-se que essa ida ao serviço possa reforçar o CS - SF como serviço de referência para essa população e possibilitar o acesso precoce para aumentar a prevenção e a promoção da saúde dos adolescentes/jovens.

A partir da análise dos relatórios elaborados pelos graduandos e das discussões realizadas nas rodas de conversa para socialização e avaliação das atividades práticas entre os estudantes, a professora e a psicóloga do CS - SF, pode-se considerar o projeto Integrar SUS - Educação como capaz de promover a aproximação qualificada dos discentes com o SUS, porque trata-se de aproximação crítica e propositiva aos desafios impostos para a sua consolidação.

Quanto à realização das oficinas, os graduandos percebem a oportunidade de realizar uma atividade em grupo como facilitadores de uma oficina de prevenção, têm a possibilidade de cooperar com um Programa do SUS, além de se sentirem participantes críticos e diretos de uma política pública de saúde em implantação.

Os jovens participantes das oficinas também avaliam o projeto com adjetivos e expressões, tais como, "legal", "produtivo", "maneiro", "informático", "deveríamos ter mais trabalhos com este aqui na escola" ou quando indagam os graduandos diretamente "quando vocês vão voltar para fazer outra atividade...", que permitem a inferência em relação à positividade da realização de oficinas no contexto escolar. De modo geral, apresentam-se bem informados sobre a epidemia 
da HIV/AIDS, conhecem as DST e informam que discutiram, em algum momento, esses assuntos nas aulas. Embora chame a atenção o fato de fazerem perguntas que remetem ao direito de receber ou não preservativos nos serviços de saúde (inclusive por serem menores de idade), mostram-se muito curiosos em relação ao preservativo feminino e ávidos por espaços de discussão para as suas experiências subjetivas, mais até que de informações básicas. Essa constatação possibilitou problematizar as três dimensões da vulnerabilidade do jovem/adolescente, a individual, a social e a programática/ institucional, no que tange à compreensão de que não basta ter informações mais acesso direto aos serviços, aos insumos mesmo antes da maioridade, ou seja, ao direito à saúde.

A aceitação também é demonstrada pelas direções das escolas e dos professores, que interpelam nos corredores para agradecer e reforçar a importância desse trabalho. Talvez nisso repouse a excelente recepção que esse grupo de graduandos, professora e psicóloga têm tido por parte dos estudantes das escolas públicas. Em quase quatro anos de implantação, apenas uma diretora não permitiu a distribuição dos preservativos durante a oficina, justificando que os pais poderiam não entender quando o estudante chegasse com os preservativos em casa e questionariam o fato de ter sido autorizado o recebimento sem a sua permissão prévia. Os argumentos de que o Estatuto da Criança e do Adolescente (ECA) defendem ações educativas que protejam os jovens e assegurem seu direito de ter acesso à saúde não foram suficientes para autorizar a distribuição. Essa ocorrência foi fruto de muitas reflexões por parte dos estudantesfacilitadores em rodas de conversa sobre os desafios de o ECA sair do papel.

No que se refere à percepção dos graduandos sobre as atividades realizadas, de modo geral, consideram que a entrada como usuário-SUS direto no aconselhamento é uma experiência muito importante, porque desmistifica o SUS, com narrativas como as apresentadas a seguir: "nunca imaginei que o SUS poderia ter um atendimento desse nível", "muitos aspectos sobre DST e HIV eu já sabia, mas me surpreendi, porque há sempre coisas para aprender". Os graduandos, ao longo do semestre, apresentam maior riqueza nas reflexões e amadurecimento das noções de prevenção e de promoção de saúde, considerando as repercussões de lidar com a sexualidade como pessoa e como futuro profissional, quando na inserção como usuário-SUS e como facilitadores das oficinas.

Outro aspecto muito importante para o êxito do Integrar SUS - Educação foi a existência do estágio curricular implantado em 2009 e da gestão participativa na UBS - SF, na qual todos os profissionais, inclusive os estagiários e a supervisora, tiveram a possibilidade de refletir sobre suas práticas e papéis com o entendimento da missão quanto ao SUS. Acredita-se que a gestão participativa só seja possível porque há a sensibilidade da gestão da unidade no que se refere ao programa/ parceria que certamente está relacionada à própria formação do gestor em saúde pública/ coletiva.

Não é incomum que a implantação de projetos dessa natureza não tenha sucesso quando o gestor não entende que ao SUS cabe ordenar a formação dos profissionais de saúde e que essa é uma tarefa de corresponsabilidade de gestores, profissionais, estudantes e professores.

Por fim, a consolidação do Integrar/SUS Educação, com o apoio do Programa de Reorientação da Formação dos Profissionais de Saúde (Pró-Saúde), fortaleceu a integração ensino-serviço-comunidade, possibilitando a implantação do estágio em Psicologia e da Atenção na Saúde Básica para os estudantes CPL em Psicologia no CS - SF. 


\section{Últimas considerações: desafios e perspectivas}

A parceria para realização das oficinas de prevenção e a experiência de entrada dos estudantes como usuários do SUS trouxe para o referido serviço repercussões positivas, visto que a equipe de saúde teve oportunidade de refletir e de discutir sobre o atendimento ao público específico dos estudantes, o que não ocorre usualmente na rotina do aconselhamento e da testagem para DST/HIV-AIDS. Inicialmente, os profissionais acreditavam que os estudantes, por serem universitários, detinham uma grande quantidade de informação, e que o aconselhamento desses estudantes deveria ser feito de uma forma mais rápida e sem o incentivo para realizar a testagem pelo SUS.

Muitas discussões foram realizadas, na sala de aula e nas reuniões técnicas entre os profissionais e a gestão, sobre a presença dos estudantes no CS - SF, resgatando a noção de saúde como direito e a importância dos princípios do SUS (universalidade, integralidade e equidade, entre outros) como orientadores das práticas oferecidas.

Por sua vez, os estudantes comentavam que nunca tinham sido usuários diretos do SUS, apesar de se darem conta, na parte teórica da disciplina, de que todos os brasileiros são seus dependentes, porque contam com o trabalho das vigilâncias epidemiológica e sanitária, por exemplo. Não imaginavam que o SUS pudesse oferecer esse tipo de serviço e com uma alta qualidade. Também relataram que muitas informações oferecidas durante o aconselhamento para testagem foram importantes para o conhecimento e o aprofundamento sobre as DST/AIDS e úteis para o desenvolvimento do papel de facilitadores nas oficinas.

Com essa prática, os estudantes colocaram a dimensão afetivo-sexual em pauta e refletiram sobre as representações sociais em torno de serem adultos-jovens, portanto, com risco de infecção em HIV e a perspectiva de atuarem futuramente na prevenção e na promoção da saúde, que supõe mudança de hábitos e de comportamentos. Sá e Tanaka (2002) problematizam bem essa questão de os trabalhadores no campo da prevenção em DST/HIV/AIDS não fazerem, na vida pessoal, sexo protegido e não conseguirem lidar com o cotidiano das questões de gênero e dos aspectos socioculturais, por exemplo, que interferem nos modos de organização da vida de todos. 


\section{Monica Lima de Jesus}

Psicologa, Doutora em Saúde Coletiva (ISC/UFBA), Professora Adjunto do Instituto de Psicologia (IPS/UFBA), Universidade Federal da Bahia, BA - Brasil.

E-mail: molije@hotmail.com

\section{Manuela Brito dos Santos}

Graduação em psicologia, Mestranda em Psicologia UFBA, Psicologa, Secretaria Municipal de Saúde - Salvador, Bahia - BA - Brasil.

E-mail:manuela_brito@hotmail.com

\section{Alice da Silva Ribeiro Firmino}

Mestre em Saúde Coletiva, Gerente do Centro de Saúde São Francisco (SMS), Professora Substituta UFBA, Professora

Faculdade Dom Pedro II - Assistente Social SESAB, Bahia - BA - Brasil.

E-mail: alicefirmino@hotmail.com

\section{Endereço para envio de correspondência:}

Rua Fonte do Boi, n. 203, ap. 26. Rio Vermelho, Salvador, Bahia - BA CEP: 41490-360

Recebido 21/1/2011, Aprovado 12/8/2011. 


\section{Referências}

Afonso, A., Abade, F., Akerman, D., Coelho, C., Medrado, K., Paulino, J., \& Pimenta, S. (2003). Oficinas em dinâmica de grupo na área da saúde. Belo Horizonte: Edições Campo Social.

Ayres, J. R. C. M., França-Júnior, I. F., Calazans, G. J., \& SallettiFilho, H. C. S. (2003). O conceito de vulnerabilidade e as práticas de saúde: novas perspectivas e desafios. In D.

Czeresnia, \& C. Freitas, (Orgs.), Promoção da saúde: conceitos, reflexões, tendências (pp. 117-140). Rio de Janeiro: Fiocruz.

Bastos, V., \& Achcar, R. (1994). Dinâmica profissional e formação do psicólogo: uma perspectiva de integração. In Conselho Federal de Psicologia. Psicólogo brasileiro: práticas emergentes e desafios para a formação (2a ed., pp. 245-271). São Paulo: Casa do Psicólogo.

Boarini, M. (1993). Unidades Básicas de Saúde: uma extensão da escola pública? Tese de doutorado, Curso de Pós-Graduação em Psicologia, Instituto de Psicologia, Universidade de São Paulo.

Boarini, M. (1996). A formação (necessária) do psicólogo para atuar na saúde pública. Revista Psicologia em Estudo, 1, 93-132.

Brasil. (jan./jun. de 2010). Ministério da Saúde - Secretária de Vigilância em Saúde - Departamento de DST, AIDS e Hepatites Virais. Boletim Epidemiológico Aids e DST, 7(01). Recuperado em 25 de junho de 2011. de http://www.aids. gov.br/publicacao/boletim-epidemiologico-2010.

Brasil. (2003). Ministério da Saúde. Manual de Aconselhamento em DST/HIV na Atenção Básica. Recuperado em 22 de março de 2007 de www.aids.gov.br.

Brasil. (2005). Ministério da Saúde. Saúde e prevenção nas escolas: atitude pra curtir a vida. Brasília, DF: Ministério de Saúde, UNESCO, \& UNICEF. Recuperado em 22 março 2009 de http://www.aids.gov.br.

Carvalho, D., \& Yamamoto, T. (1999). Psicologia e saúde: uma análise da estruturação de um novo campo teórico-prático. Psico, 30(1), 5-28.

Centro de Referência em Políticas Públicas e Psicologia (CREPOP). (2008, dez.). Referências técnicas para a prática do (a) psicólogo (a) nos programas de DST e AIDS. Brasília DF. Recuperado em 20 de novembro de 2010 de www.pol.org.br.
Departamento de DST, AIDS e Hepatites Virais. (2008). Pesquisa de conhecimentos, atitudes e práticas relacionadas às DSTs e AIDS da população brasileira de 15 a 64 anos de idade. IBOPE. Recuperado em 25 de junho de 2011 de http://www.aids.gov. br/publicacao/pcap-2008.

Dimenstein, M. (1998). O psicólogo nas unidades básicas de saúde: desafios para a formação e atuação profissionais. Estudos de Psicologia, 3(1), 53-81.

Lima, M. (2005a). Atuação psicológica em serviços públicos de saúde de Salvador: do ponto de vista dos psicólogos. Tese de doutorado, Universidade Federal da Bahia. Programa de Pós-Graduação do Instituto de Saúde Coletiva.

Lima, M. (ago./dez. de ?). As diretrizes curriculares nacionais para a graduação em Psicologia em três poemas modernistas. Jornal CRP-03, 2, 11-12. Recuperado em 28 outubro 2010 de www.crpsp.org.br.

Lima, M. (2005b, dez.). Atuação psicológica coletiva: uma trajetória profissional em unidade básica de saúde. Psicol. Estud., 10( 3), 431-440.

Lo Bianco A., Bastos, A., Nunes, M., \& Silva, R. (1994). Concepções e atividades emergentes na psicologia clínica: implicações para a formação. In Conselho Federal de Psicologia. Psicólogo brasileiro: práticas emergentes e desafios para a formação (2a ed., pp. 7-79). São Paulo: Casa do Psicólogo.

Macêdo, R., Heloani, K., \& Cassilato, R. (2010). O psicólogo como trabalhador assalariado: setor de inserção, locais, atividades e condições de trabalho. In Bastos, A., \& Gondim, S. (Orgs.), O trabalho do psicólogo brasileiro (pp. 131-150). Porto Alegre: Artmed.

Sá, S., \& Tanaka, O. (2002, jun.). O saber e a prevenção no trabalho e na vida: representações de profissionais de saúde que trabalham com HIV/AIDS. Psicol.: Ciênc. e Prof., 22( 2), 45-70.

Silva, R. (1992). A formação em psicologia para o trabalho na saúde pública. In F. Campos (Org.), Psicologia e saúde: repensando práticas (pp. 25-40). São Paulo: Hucitec.

Spink, M. J. P. (Org.). (2007). A psicologia em diálogo com o SUS: prática profissional e produção acadêmica. São Paulo: Casa do Psicólogo. 